\title{
The Coarse Baum-Connes Conjecture for Spaces Which Admit a Uniform Embedding into Hilbert Space*
}

\author{
GuOliang Yu
}

July 1, 1998

\section{Introduction}

Let $\Gamma$ be a metric space; let $H$ be a separable and infinite dimensional Hilbert space. A map $f: \Gamma \rightarrow H$ is said to be a uniform embedding [10] if there exist non-decreasing functions $\rho_{1}$ and $\rho_{2}$ from $\mathbb{R}_{+}=[0, \infty)$ to $\mathbb{R}$ such that

(1) $\rho_{1}(d(x, y)) \leq\|f(x)-f(y)\| \leq \rho_{2}(d(x, y))$ for all $x, y \in \Gamma$;

(2) $\lim _{r \rightarrow+\infty} \rho_{i}(r)=+\infty$ for $i=1,2$.

The main purpose of this paper is to prove the following result:

Theorem 1.1. Let $\Gamma$ be a discrete metric space with bounded geometry. If $\Gamma$ admits a uniform embedding into Hilbert space, then the coarse Baum-Connes conjecture holds for $\Gamma$.

Recall that a discrete metric space $\Gamma$ is said to have bounded geometry if $\forall r>0$, $\exists N(r)>0$ such that the number of elements in $B(x, r)$ is at most $N(r)$ for all $x \in \Gamma$, where $B(x, r)=\{y \in \Gamma: d(y, x) \leq r\}$. Every finitely generated group, as a metric space with a word length metric, has bounded geometry.

Corollary 1.2. Let $\Gamma$ be a finitely generated group. If $\Gamma$, as a metric space with a word length metric, admits a uniform embedding into Hilbert space, and its classifying space B $\Gamma$

*Partially supported by the National Science Foundation 
has the homotopy type of a finite CW complex, then the strong Novikov conjecture holds for $\Gamma$, i.e. the index map from $K_{*}(B \Gamma)$ to $K_{*}\left(C_{r}^{*}(\Gamma)\right)$ is injective.

Corollary 1.2 follows from Theorem 1.1 and the descent principle [22]. By index theory, the strong Novikov conjecture implies the Novikov conjecture on the homotopy invariance of higher signatures (c.f. [8] for an excellent survey of the Novikov conjecture). The class of finitely generated groups which admit a uniform embedding into Hilbert space contains a subclass of groups which includes Gromov's word hyperbolic groups and amenable groups, and is closed under semi-direct product (c.f. Proposition 2.6). In general, it is an open question if every finitely generated group (or separable metric space) admits a uniform embedding into Hilbert space ([10], page 218, [11], page 67). However, it can be easily proved that every metric space admits a uniform embedding into a Banach space (c.f. Proposition 2.7).

This work is inspired by Gromov's deep questions concerning uniform embedding into Hilbert space $([10],[11])$ and by the remarkable work of Higson and Kasparov on the Baum-Connes conjecture [14].

\section{Uniform embeddings into Hilbert space and property $A$}

In this section, we shall introduce the concept of property A for metric spaces. We prove that metric spaces with property A admit a uniform embedding into Hilbert space. The class of finitely generated groups with property A, as metric spaces with word length metrics, includes word hyperbolic groups and amenable groups, and is closed under semidirect product. We also show that every metric space admits a uniform embedding into a Banach space.

Definition 2.1. A discrete metric space $\Gamma$ is said to have property $\mathrm{A}$ if for any $r>0$, $\varepsilon>0$, there exist a family of finite subsets $\left\{A_{\gamma}\right\}_{\gamma \in \Gamma}$ of $\Gamma \times \mathbb{N}$ ( $\mathbb{N}$ is the set of all natural numbers ) such that

(1) $(\gamma, 1) \in A_{\gamma}$ for all $\gamma \in \Gamma$; 
(2) $\frac{\#\left(A_{\gamma}-A_{\gamma^{\prime}}\right)+\#\left(A_{\gamma^{\prime}}-A_{\gamma}\right)}{\#\left(A_{\gamma} \cap A_{\gamma^{\prime}}\right)}<\varepsilon$ for all $\gamma, \gamma^{\prime} \in \Gamma$ satisfying $d\left(\gamma, \gamma^{\prime}\right) \leq r$, where, for each finite set $A, \# A$ is the number of elements in $A$;

(3) $\exists R>0$ such that if $(x, m) \in A_{\gamma},(y, n) \in A_{\gamma}$ for some $\gamma \in \Gamma$, then $d(x, y) \leq R$.

Notice that property $\mathrm{A}$ is invariant under quasi-isometry. In the case of a finitely generated group, property A does not depend on the choice of the word length metric.

Theorem 2.2. If a discrete metric space $\Gamma$ has property $A$, then $\Gamma$ admits a uniform embedding into Hilbert space.

Proof. Let

$$
H=\bigoplus_{k=1}^{\infty} \ell^{2}(\Gamma \times \mathbb{N})
$$

By the definition of property A, there exist a family of finite subsets $\left\{A_{\gamma}^{(k)}\right\}_{\gamma \in \Gamma}$ such that (1) $(\gamma, 1) \in A_{\gamma}^{(k)}$ for all $\gamma \in \Gamma$;

(2) $\exists R_{k}>0$ such that if $(x, m) \in A_{\gamma}^{(k)},(y, n) \in A_{\gamma}^{(k)}$ for some $k$ and $\gamma \in \Gamma$, then $d(x, y) \leq R_{k}$

$$
\left\|\frac{\chi_{A_{\gamma}^{(k)}}}{\left(\# A_{\gamma}^{(k)}\right)^{1 / 2}}-\frac{\chi_{A_{\gamma^{\prime}}^{(k)}}}{\left(\# A_{\gamma^{\prime}}^{(k)}\right)^{1 / 2}}\right\|_{l^{2}(\Gamma \times \mathbb{N})}<\frac{1}{2^{k}}
$$

for all $\gamma, \gamma^{\prime} \in \Gamma$ satisfying $d\left(\gamma, \gamma^{\prime}\right) \leq k$ and $k \in \mathbb{N}$, where $\chi_{A_{\gamma}^{(k)}}$ is the characteristic function of $A_{\gamma}^{(k)}$.

Fix $\gamma_{0} \in \Gamma$. Define $f: \Gamma \rightarrow H$ by:

$$
f(\gamma)=\bigoplus_{k=1}^{\infty}\left(\frac{\chi_{A_{\gamma}^{(k)}}}{\left(\# A_{\gamma}^{(k)}\right)^{1 / 2}}-\frac{\chi_{A_{\gamma_{0}}^{(k)}}}{\left(\# A_{\gamma_{0}}^{(k)}\right)^{1 / 2}}\right)
$$

One can easily check that $f$ is a uniform embedding. QED

Example 2.3: Let $\Gamma$ be a finitely generated amenable group. $\forall r>0,0.5>\varepsilon>0$, there exists a finite subset $F$ of $\Gamma$ such that

$$
\frac{\#(F g-F)+\#(F-F g)}{\# F}<\varepsilon / 10 \quad \text { if } \quad d(g, 1) \leq r
$$


where $d$ is the word length metric. Set $A_{\gamma}=\{(x, 1) \in \Gamma \times \mathbb{N}: x \in \gamma F\}$. It is easy to see that $\left\{A_{\gamma}\right\}_{\gamma \in \Gamma}$ satisfies the conditions of property A.

Hence amenable groups admit a uniform embedding into Hilbert space. More generally, groups acting properly and isometrically on Hilbert space admit a uniform embedding into Hilbert space [2]. However, the class of groups admitting a uniform embedding into Hilbert space is much larger than the class of groups acting properly and isometrically on Hilbert space since infinite property $\mathrm{T}$ groups can not act properly and isometrically on Hilbert space.

Example 2.4: Let $F_{2}$ be the free group of two generators. Let $T$ be the tree associated to $F_{2}$, where the set of all vertices of $T$ is $F_{2}$. Endow $T$ with the simplicial metric. Fix a geodesic ray $\gamma_{0}$ on $T$. For each $g \in F_{2}$, there exists a unique geodesic ray $\gamma_{g}$ on $T$ starting from $g$ such that $\gamma_{g} \cap \gamma_{0}$ is a non-empty geodesic ray. For each natural number $N$, define

$$
A_{g}=\left\{(x, 1) \in F_{2} \times \mathbb{N}, x \in \gamma_{g}, d(x, g) \leq N\right\}
$$

for all $g \in F_{2}$.

Given $r>0, \varepsilon>0$, it is not difficult to see that there exists a large $N$ such that $\left\{A_{g}\right\}_{g \in F_{2}}$ satisfies the conditions of property A.

Example 2.5: Let $M$ be a compact Riemannian manifold with negative curvature, let $\Gamma=\pi_{1}(M)$, the fundamental group of $M$. Fix a point $x_{0} \in \widetilde{M}$, the universal cover of $M$, and a geodesic ray (with unit speed) $\gamma_{0}$ on $\widetilde{M}$ starting from $x_{0}$. For each $g \in \Gamma$, let $\gamma_{g}$ be a geodesic ray (with unit speed) on $\widetilde{M}$ starting from $g x_{0}$ such that $\gamma_{g}$ is asymptotic to $\gamma_{0}$. For each natural number $N$, we define

$$
B_{g}=\left\{x \in \widetilde{M}, d\left(x, \gamma_{g}(t)\right)<1 \text { for some } t \in[0, N]\right\}
$$

Let $F$ be a fundamental domain in $\widetilde{M}$. For each $\delta_{0}>0, \delta_{1}>0$, there exists a natural number $k$ such that if volume $\left(g^{\prime} F \cap B_{g}\right) \geq \delta_{0}$ for some $g, g^{\prime} \in \Gamma$, then there exists an 
integer $\ell_{g^{\prime}, g}$ satisfying

$$
\left|\operatorname{volume}\left(g^{\prime} F \cap B_{g}\right)-\frac{\ell_{g^{\prime}, g}}{k}\right|<\delta_{1}
$$

We define

$$
A_{g}=\left\{\left(g^{\prime}, n\right): \text { volume }\left(g^{\prime} F \cap B_{g}\right) \geq \delta_{0}, 1 \leq n \leq \ell_{g^{\prime}, g}\right\} \subseteq \Gamma \times \mathbb{N}
$$

Using comparison theorems in Riemannian geometry and the negative curvature property, it is not difficult to verify that if $N$ is large enough, $\delta_{0}$ and $\delta_{1}$ are small enough, then $\left\{A_{g}\right\}_{g \in \Gamma}$ satisfies the conditions of property A.

More generally, one can show that word hyperbolic groups have property A by similar argument using constructions from [24].

Proposition 2.6. Let $\Gamma_{1}$ and $\Gamma_{2}$ be two finitely generated groups with property $A$ (as metric spaces with word length metrics). If $\Gamma_{1}$ acts on $\Gamma_{2}$ by automorphisms, then the semi-direct product $\Gamma_{2} \rtimes \Gamma_{1}$ has property $A$.

Proof. Given $r>0, \varepsilon>0$. For each $r_{1}>0, \varepsilon_{1}>0, r_{2}>0, \varepsilon_{2}>0$, let $\left\{A_{\gamma}^{(1)}\right\}_{\gamma \in \Gamma_{1}}$ and $\left\{A_{\gamma}^{(2)}\right\}_{\gamma \in \Gamma_{2}}$ be respectively as in the definition of property $\mathrm{A}$ for $\Gamma_{1}$ with respect to $r_{1}$ and $\varepsilon_{1}$, and for $\Gamma_{2}$ with respect to $r_{2}$ and $\varepsilon_{2}$. Set

$$
f\left(x \cdot y,\left(\gamma_{1} \cdot \gamma_{2},(m, n)\right)\right)=\chi_{A_{x}^{(1)}}\left(\gamma_{1}, m\right) \chi_{A_{\gamma_{1}^{-1} x y x^{-1} \gamma_{1}}^{(2)}}\left(\gamma_{2}, n\right),
$$

where $m, n \in \mathbb{N}, x \in \Gamma_{1}, y \in \Gamma_{2}, x \cdot y \in \Gamma_{2} \rtimes \Gamma_{1}, \gamma_{1} \in \Gamma_{1}, \gamma_{2} \in \Gamma_{2}, \gamma_{1} \cdot \gamma_{2} \in \Gamma_{2} \rtimes \Gamma_{1}$, and, for each set $A, \chi_{A}$ is the characteristic function of $A$.

Let $h$ be a bijective map from $\mathbb{N}$ to $\mathbb{N} \times \mathbb{N}$ such that $h(1)=(1,1)$. For each $\gamma=x \cdot y \in$ $\Gamma_{2} \rtimes \Gamma_{1}$, we define

$$
A_{\gamma}=\left\{\left(\gamma_{1} \cdot \gamma_{2}, n\right) \in\left(\Gamma_{2} \rtimes \Gamma_{1}\right) \times \mathbb{N}: f\left(x \cdot y,\left(\gamma_{1} \cdot \gamma_{2}, h(n)\right)\right) \neq 0\right\}
$$

Now it is straightforward to verify that if $r_{i}$ and $\varepsilon_{i}(i=1,2)$ are chosen appropriately, then $\left\{A_{\gamma}\right\}_{\gamma \in \Gamma_{2} \rtimes \Gamma_{1}}$ satisfies the conditions of property A. QED 
In general, it is an open question if every separable discrete metric space admits a uniform embedding [10]. The following result gives us some hope that it might be true.

Proposition 2.7. Every discrete metric space $\Gamma$ admits a uniform embedding into a Banach space.

Proof. Fix $x_{0} \in \Gamma$. Define a map $f: \Gamma \rightarrow \ell^{\infty}(\Gamma)$ by:

$$
(f(x))(\gamma)=d(\gamma, x)-d\left(\gamma, x_{0}\right)
$$

for all $x, \gamma \in \Gamma$.

It is not difficult to see that $f$ is a uniform embedding. QED

We remark that if $\Gamma$ is separable, then there exists a separable Banach space into which $\Gamma$ has a uniform embedding.

\section{The coarse Baum-Connes conjecture}

In this section, we shall briefly recall the coarse Baum-Connes conjecture and its applications.

Let $M$ be a proper metric space (a metric space is called proper if every closed ball is compact). Let $H_{M}$ be a separable Hilbert space equipped with a faithful and nondegenerate representation of $C_{0}(M)$ whose range contains no nonzero compact operator.

Definition 3.1: (1) The support of a bounded linear operator $T: H_{M} \rightarrow H_{M}$ is the complement of the set of points $\left(m, m^{\prime}\right) \in M \times M$ for which there exists $f$ and $f^{\prime}$ in $C_{0}(M)$ such that

$$
f^{\prime} T f=0, \quad f(m) \neq 0 \quad \text { and } \quad f^{\prime}\left(m^{\prime}\right) \neq 0
$$

(2) A bounded operator $T: H_{M} \rightarrow H_{M}$ has finite propagation if $\sup \left\{d\left(m, m^{\prime}\right)\right.$ : $\left.\left(m, m^{\prime}\right) \in \operatorname{supp}(T)\right\}<\infty$;

(3) A bounded operator $T: H_{M} \rightarrow H_{M}$ is locally compact if the operators $f T$ and $T f$ are compact for all $F \in C_{0}(M)$. 
Definition 3.2: The Roe algebra $C^{*}(M)$ is the operator norm closure of the $*$-algebra of all locally compact, finite propagation operators acting on $H_{M}$.

$C^{*}(M)$ is independent of the choice of $H_{M}$ (up to $*$ isomorphism). In particular, we can choose $H_{M}$ to be $l^{2}(Z) \otimes H_{0}$, where $Z$ is a countable dense subset of $M, H_{0}$ is a separable and infinite dimensional Hilbert space, and $C_{0}(M)$ acts on $H_{M}$ by: $f(g \otimes h)=f g \otimes h$ for all $f \in C_{0}(M), g \in l^{2}(Z), h \in H_{0}$ ( $f$ acts on $l^{2}(Z)$ by pointwise multiplication $)$. Such a choice of $H_{M}$ will be useful later on in this paper.

Let $\Gamma$ be a locally finite discrete metric space (a metric space is called locally finite if every ball contains finitely many elements).

Definition 3.3: For each $d \geq 0$, the Rips complex $P_{d}(\Gamma)$ is the simplicial polyhedron where the set of all vertices is $\Gamma$, and a finite subset $\left\{\gamma_{0}, \ldots, \gamma_{n}\right\} \subseteq \Gamma$ spans a simplex iff $d\left(\gamma_{i}, \gamma_{j}\right) \leq d$ for all $0 \leq i, j \leq n$.

Endow $P_{d}(\Gamma)$ with the spherical metric. Recall that the spherical metric is the maximal metric whose restriction to each simplex is the metric obtained by identifying the simplex with a half (unit) sphere endowed with the standard Riemannian metric. The distance of a pair of points in different connected components of $P_{d}(\Gamma)$ is defined to be infinity. Use of the spherical metric is necessary in Section 4 to avoid certain pathological phenomena when $d$ goes to infinity.

Conjecture 3.4. (The Coarse Baum-Connes Conjecture) If $\Gamma$ is a discrete metric space with bounded geometry, then the index map from $\lim _{d \rightarrow \infty} K_{*}\left(P_{d}(\Gamma)\right)$ to $\lim _{d \rightarrow \infty} K_{*}\left(C^{*}\left(P_{d}(\Gamma)\right)\right)$ is an isomorphism, where $K_{*}\left(P_{d}(\Gamma)\right)=K K_{*}\left(C_{0}\left(P_{d} \Gamma\right), \mathbb{C}\right)$ is the locally finite $K$-homology group of $P_{d}(\Gamma)$.

This conjecture is false if the bounded geometry condition is dropped [27]. In the case of a finitely generated group, the coarse Baum-Connes conjecture for the group as a metric space with a word length metric implies the Novikov conjecture if the group has a finite $C W$ complex as its classifying space [22]. The coarse Baum-Connes conjecture also 
has consequences on the positive scalar curvature problem and the zero-in-the spectrum problem [21].

\section{The localization algebra}

The localization algebra introduced in [26] will play an important role in the proof of our main result. For the convenience of the readers, we shall briefly recall its definition and its relation with K-homology.

Let $M$ be a proper metric space.

Definition 4.1: The localization algebra $C_{L}^{*}(M)$ is the norm-closure of the algebra of all uniformly bounded and uniformly norm-continuous functions $f:[0, \infty) \rightarrow C^{*}(M)$ such that

$$
\sup \left\{d\left(m, m^{\prime}\right):\left(m, m^{\prime}\right) \in \operatorname{supp}(f(t))\right\} \rightarrow 0
$$

as $t \rightarrow \infty$.

There exists a local index map $([26])$ :

$$
\operatorname{ind}_{L}: K_{*}(M) \rightarrow K_{*}\left(C_{L}^{*}(M)\right)
$$

The evaluation homomorphism $e$ from $C_{L}^{*}(M)$ to $C^{*}(M)$ is defined by:

$$
e(f)=f(0)
$$

If $\Gamma$ is a locally finite discrete metric space, we have the following commuting diagram:

$$
\begin{gathered}
\lim _{d \rightarrow \infty} K_{*}\left(P_{d}(\Gamma)\right) \\
\operatorname{ind}_{L} \swarrow \\
\lim _{d \rightarrow \infty} K_{*}\left(C_{L}^{*}\left(P_{d}(\Gamma)\right)\right) \stackrel{e_{*}}{\longrightarrow} \lim _{d \rightarrow \infty} K_{*}\left(C^{*}\left(P_{d}(\Gamma)\right)\right) .
\end{gathered}
$$


Theorem 4.2. ([26]) If $\Gamma$ has bounded geometry, then ind ${ }_{L}$ is an isomorphism.

The above theorem implies that in order to prove the coarse Baum-Connes conjecture, it is enough to show that

$$
e_{*}: \lim _{d \rightarrow \infty} K_{*}\left(C_{L}^{*}\left(P_{d}(\Gamma)\right)\right) \rightarrow \lim _{d \rightarrow \infty} K_{*}\left(C^{*}\left(P_{d}(\Gamma)\right)\right)
$$

is an isomorphism.

\section{Twisted Roe algebras and twisted localization algebras}

In this section, we shall introduce certain twisted Roe algebras and twisted localization algebras. These algebras will play important roles in the proof of our main result.

We shall first recall an algebra associated to an infinite dimensional Euclidean space introduced by Higson, Kasparov and Trout [15]. Let $V$ be a countably infinite dimensional Euclidean space. Denote by $V_{a}, V_{b}$, so on, the finite dimensional, affine subspaces of $V$. Denote by $V_{a}^{0}$ the finite dimensional linear subspace of $V$ consisting of differences of elements in $V_{a}$. let $\mathcal{C}\left(V_{a}\right)$ be the $\mathbb{Z} / 2$-graded $C^{*}$-algebra of continuous functions from $V_{a}$ into the complexified Clifford algebra of $V_{a}^{0}$ which vanish at infinity.

Let $\mathcal{S}=C_{0}(\mathbb{R})$, graded according to even and odd functions. Let $\mathcal{A}\left(V_{a}\right)$ be the graded tensor product of $\mathcal{S}$ with $\mathcal{C}\left(V_{a}\right)$.

If $V_{a} \subset V_{b}$, we have a decomposition:

$$
V_{b}=V_{b a}^{0}+V_{a}
$$

where $V_{b a}^{0}$ is the orthogonal complement of $V_{a}^{0}$ in $V_{b}^{0}$. For each $v_{b} \in V_{b}$, we have a corresponding decomposition: $v_{b}=v_{b a}+v_{a}$, where $v_{b a} \in V_{b a}^{0}$ and $v_{a} \in V_{a}$.

Definition 5.1: (1) If $V_{a} \subset V_{b}$, denote by $C_{b a}$ the Clifford algebra-valued function on $V_{b}$ which maps $v_{b}$ to $v_{b a} \in V_{b a}^{0} \subset \operatorname{Cliff}\left(V_{b}^{0}\right)$. Define a homomorphism $\beta_{b a}: \mathcal{A}\left(V_{a}\right) \rightarrow \mathcal{A}\left(V_{b}\right)$, by

$$
\beta_{b a}(f \widehat{\otimes} h)=f\left(X \widehat{\otimes} 1+1 \widehat{\otimes} C_{b a}\right)(1 \widehat{\otimes} h)
$$


for all $f \in \mathcal{S}$ and $h \in \mathcal{C}\left(V_{a}\right)$, where $X$ is the function of multiplication by $x$ on $\mathbb{R}$, considered as a degree one, unbounded multiplier of $\mathcal{S}$.

(2) We define a $C^{*}$-algebra $\mathcal{A}(V)$ by:

$$
\mathcal{A}(V)=\underset{\lim }{\longrightarrow} \mathcal{A}\left(V_{a}\right)
$$

where the direct limit is over the directed set of all finite dimensional affine subspaces $V_{a} \subset V$, using the homomorphism $\beta_{b a}$ in (1).

Let $\Gamma$ be a discrete metric space with bounded geometry. Assume that $\Gamma$ admits a uniform embedding $f: \Gamma \rightarrow H$, where $H$ is a separable Hilbert space. By the bounded geometry property, there exist a family of Euclidean spaces $\{W(x)\}_{x \in \Gamma}$ such that $W(x)$ is dense in $H$ for all $x \in \Gamma$, for each $n \in \mathbb{N}$ there exists a finite dimensional Euclidean subspace $W_{n}(x) \subseteq W(x)$ for which

(1) $W_{n}(x) \subseteq W_{n+1}(x)$ for all $n \in \mathbb{N}, x \in \Gamma$, and $W(x)=\bigcup_{n \in \mathbb{N}} W_{n}(x)$ for all $x \in \Gamma$;

(2) $\forall n \in \mathbb{N}, \exists d_{n}>0$ such that $\operatorname{dim} W_{n}(x) \leq d_{n}$ for all $x \in \Gamma$;

(3) $\forall r>0, \exists n_{r}>0$ such that $U_{y, x} W_{n}(x) \subseteq W_{n+1}(y)$ for all $x, y \in \Gamma$ satisfying $d(x, y) \leq r$ and all $n>n_{r}$, where $U_{y, x} h=h+f(y)-f(x)$ for all $h \in H$.

Condition (3) implies that there exists an Euclidean subspace $V$ of $H$ such that $W(x)=V$ for all $x \in \Gamma$.

For every $x \in P_{d}(\Gamma)$, write $x=\sum_{\substack{\gamma \in \Gamma \\ c_{\gamma}>0}} c_{\gamma} \gamma$. Define $W_{n}(x)$ to be the Euclidean subspace of $H$ spanned by $W_{n}(\gamma)$ for all $\gamma$ such that $c_{\gamma}>0$. We extend $f$ to $P_{d}(\Gamma)$ by:

$$
f(x)=\sum c_{\gamma} f(\gamma)
$$

We define the affine isometry $U_{y, x}: V \rightarrow V$ for every pair $x, y \in P_{d}(\Gamma)$ by:

$$
U_{y, x} h=h+f(y)-f(x)
$$

for all $h \in V . U_{y, x}$ induces a $*$ isomorphism from $\mathcal{A}(V)$ to $\mathcal{A}(V)$ denoted by:

$$
a \rightarrow U_{y, x}(a)
$$


Throughout the rest of this paper, $\mathbb{R}_{+} \times H$ is endowed with the weakest topology for which the projection to $H$ is weakly continuous and the function $t^{2}+\|h\|^{2}$ is continuous $\left((t, h) \in \mathbb{R}_{+} \times H\right)$ (c.f. [14]). The center of $\mathcal{A}(V)$ contains $C_{0}\left(\mathbb{R}_{+} \times H\right)$, where $C_{0}\left(\mathbb{R}_{+} \times H\right)$ is the algebra of all continuous functions on $\mathbb{R}_{+} \times H$ which vanish at infinity. The support of an element $a \in \mathcal{A}(V)$ is defined to be the complement (in $\mathbb{R}_{+} \times H$ ) of all $(t, h)$ for which there exists $f \in C_{0}\left(\mathbb{R}_{+} \times H\right)$ such that $a f=0$ and $f(t, h) \neq 0$.

Choose a countable dense subset $\Gamma_{d}$ of $P_{d}(\Gamma)$ for each $d>0$ such that $\Gamma_{d_{1}} \subseteq \Gamma_{d_{2}}$ if $d_{2} \geq d_{1}$

Let $C_{\text {alg }}^{*}\left(P_{d}(\Gamma), \mathcal{A}\right)$ be the set of all functions $T$ on $\Gamma_{d} \times \Gamma_{d}$ such that

(1) $\exists$ an integer $N$ such that $T(x, y) \in\left(\beta_{N}(x)\right)\left(\mathcal{A}\left(W_{N}(x)\right) \widehat{\otimes} K\right) \subseteq \mathcal{A}(V) \widehat{\otimes} K$ for all $x, y \in \Gamma_{d}$, where $\beta_{N}(x): \mathcal{A}\left(W_{N}(x)\right) \widehat{\otimes} K \rightarrow \mathcal{A}(V) \widehat{\otimes} K$, is the $*$ homomorphism associated to the inclusion of $W_{N}(x)$ into $V$, and $K$ is the algebra of compact operators;

(2) $\exists M>0$ and $L>0$ such that $\|T(x, y)\| \leq M$ for all $x, y \in \Gamma_{d}$, and for each $y \in \Gamma_{d}$, $\#\{x: T(x, y) \neq 0\} \leq L, \#\{x: T(y, x) \neq 0\} \leq L$

(3) $\exists r_{1}>0$ and $r_{2}>0$ such that

(a) if $d(x, y)>r_{1}$, then $T(x, y)=0$;

(b) $\operatorname{support}(T(x, y)) \subseteq B\left(r_{2}\right)$ for all $x, y \in \Gamma_{d}$, where $B\left(r_{2}\right)=\left\{(s, h) \in \mathbb{R}_{+} \times H: s^{2}+\right.$ $\left.\|h\|^{2}<r_{2}^{2}\right\}$

(4) $\exists c>0$ such that $D_{Y}\left(T_{1}(x, y)\right)$ exists in $\mathcal{A}\left(W_{N}(x)\right) \widehat{\otimes} K$, and $\left\|D_{Y}\left(T_{1}(x, y)\right)\right\| \leq c$ for all $x, y \in \Gamma_{d}$ and $Y=(s, h) \in \mathbb{R} \times W_{N}(x)$ satisfying $\|Y\|=\sqrt{s^{2}+\|h\|^{2}} \leq 1$, where $\left(\beta_{N}(x)\right)\left(T_{1}(x, y)\right)=T(x, y)$, and $D_{Y}\left(T_{1}(x, y)\right)$ is the derivative of the function $T_{1}(x, y)$ : $\mathbb{R} \times W_{N}(x) \rightarrow \mathcal{C}\left(W_{N}(x)\right) \widehat{\otimes} K$, in the direction of $Y$.

It will become clear in the proof of Lemma 7.3 why we require condition (4) in the above definition.

We define a product structure on $C_{a l g}^{*}\left(P_{d}(\Gamma), \mathcal{A}\right)$ by:

$$
\left(T_{1} T_{2}\right)(x, y)=\sum_{z \in \Gamma_{d}} T_{1}(x, z) U_{x, z}\left(T_{2}(z, y)\right) .
$$


Let

$$
E=\left\{\sum_{x \in \Gamma_{d}} a_{x}[x]: a_{x} \in \mathcal{A}(V) \widehat{\otimes} K, \sum_{x \in \Gamma_{d}}\left\|a_{x}\right\|^{2}<+\infty\right\} .
$$

Fix $x_{0} \in \Gamma . E$ is a Hilbert module over $\mathcal{A}(V) \widehat{\otimes} K$ :

$$
\begin{aligned}
\left\langle\sum_{x \in \Gamma_{d}} a_{x}[x], \sum_{x \in \Gamma_{d}} b_{x}[x]\right\rangle & =\sum_{x \in \Gamma_{d}}\left(U_{x_{0}, x}\left(a_{x}\right)\right)^{*}\left(U_{x_{0}, x}\left(b_{x}\right)\right), \\
\left(\sum_{x \in \Gamma_{d}} a_{x}[x]\right) a & =\sum_{x \in \Gamma_{d}} a_{x} U_{x, x_{0}}(a)[x]
\end{aligned}
$$

for all $a \in \mathcal{A}(V) \widehat{\otimes} K$.

$C_{\text {alg }}^{*}\left(P_{d}(\Gamma), \mathcal{A}\right)$ acts on $E$ by:

$$
T\left(\sum_{x \in \Gamma_{d}} a_{x}[x]\right)=\sum_{y \in \Gamma_{d}}\left(\sum_{x \in \Gamma_{d}} T(y, x) U_{y, x}\left(a_{x}\right)\right)[y],
$$

where $T \in C_{a l g}^{*}\left(P_{d}(\Gamma), \mathcal{A}\right), \sum a_{x}[x] \in E$. One can easily verify that $T$ is a module homomorphism which has an adjoint module homomorphism.

Definition 5.2: $C^{*}\left(P_{d}(\Gamma), \mathcal{A}\right)$ is the operator norm closure of $C_{\text {alg }}^{*}\left(P_{d}(\Gamma), \mathcal{A}\right)$ in $B(E)$, the $C^{*}$ algebra of all module homomorphisms from $E$ to $E$ for which there is an adjoint module homomorphism.

Let $C_{L, \text { alg }}^{*}\left(P_{d}(\Gamma), \mathcal{A}\right)$ be the set of all uniformly norm-continuous and uniformly bounded functions $g: \mathbb{R}_{+} \rightarrow C_{a l g}^{*}\left(P_{d}(\Gamma), \mathcal{A}\right)$ such that

(1) $\exists N$ such that $(g(t))(x, y) \in\left(\beta_{N}(x)\right)\left(\mathcal{A}\left(W_{N}(x)\right) \widehat{\otimes} K\right) \subseteq \mathcal{A}(V) \widehat{\otimes} K$ for all $t \in \mathbb{R}_{+}$, $x, y \in \Gamma_{d}$

(2) $\exists$ a bounded function $r(t): \mathbb{R}_{+} \rightarrow \mathbb{R}_{+}$such that $\lim _{t \rightarrow \infty} r(t)=0$ and if $d(x, y)>$ $r(t)$, then $(g(t))(x, y)=0$;

(3) $\exists R>0$ such that $\operatorname{support}((g(t))(x, y)) \subseteq B(R)$ for all $t \in \mathbb{R}_{+}, x, y \in \Gamma_{d}$, $B(R)=\left\{(s, h) \in \mathbb{R}_{+} \times H: s^{2}+\|h\|^{2}<R^{2}\right\}$

(4) $\exists C>0$ such that $\left\|D_{Y}\left(\left(g_{1}(t)\right)(x, y)\right)\right\| \leq C$ for all $t \in \mathbb{R}_{+}, x, y \in \Gamma_{d}$ and $Y \in$ $\mathbb{R} \times W_{N}(x)$ satisfying $\|Y\| \leq 1$, where $\left(\beta_{N}(x)\right)\left(\left(g_{1}(t)\right)(x, y)\right)=(g(t))(x, y)$. 
Definition 5.3: $C_{L}^{*}\left(P_{d}(\Gamma), \mathcal{A}\right)$ is the norm closure of $C_{L, a l g}^{*}\left(P_{d}(\Gamma), \mathcal{A}\right)$, where $C_{L, \text { alg }}^{*}\left(P_{d}(\Gamma), \mathcal{A}\right)$ is endowed with the norm:

$$
\|g\|=\sup _{t \in \mathbb{R}_{+}}\|g(t)\|_{C^{*}\left(P_{d}(\Gamma), \mathcal{A}\right)}
$$

\section{K-Theory of twisted Roe algebras and twisted localization algebras}

In this section, we shall study the K-theory of the twisted Roe algebras and the twisted localization algebras.

Definition 6.1: (1) The support of an element $T$ in $C_{\text {alg }}^{*}\left(P_{d}(\Gamma), \mathcal{A}\right)$ is defined to be

$$
\begin{aligned}
& \left\{(u, x) \times\left(U_{y, x}^{-1} u, y\right) \in\left(\left(\mathbb{R}_{+} \times H\right) \times \Gamma_{d}\right) \times\left(\left(\mathbb{R}_{+} \times H\right) \times \Gamma_{d}\right):\right. \\
& T(y, x) \neq 0, u \in \operatorname{supp}(T(y, x))\},
\end{aligned}
$$

where $U_{y, x}^{-1} u=\left(t, U_{y, x}^{-1} h\right)$ for $u=(t, h) \in \mathbb{R}_{+} \times H$;

(2) The support of an element $g$ in $C_{L, a l g}^{*}\left(P_{d}(\Gamma), \mathcal{A}\right)$ is defined to be $\bigcup_{t \in \mathbb{R}_{+}} \operatorname{supp}(g(t))$.

We define an equivalence relation on $\left(\mathbb{R}_{+} \times H\right) \times \Gamma_{d}$ by

$$
(u, x) \sim(v, y) \quad \text { iff } \quad v=U_{x, y} u
$$

where $u, v \in \mathbb{R}_{+} \times H$ and $x, y \in \Gamma_{d}$.

Fix $x_{0} \in \Gamma$ as in Section 5. Let $O$ be an open subset of $\mathbb{R}_{+} \times H$; let $O(d)$ be a subset of $\left(\mathbb{R}_{+} \times H\right) \times \Gamma_{d}$ defined by:

$$
O(d)=\left\{(u, x) \in\left(\mathbb{R}_{+} \times H\right) \times \Gamma_{d}:(u, x) \sim\left(v, x_{0}\right) \quad \text { for some } \quad v \in O\right\} .
$$

Let $C_{\text {alg }}^{*}\left(P_{d}(\Gamma), \mathcal{A}\right)_{O}$ be the subalgebra of $C_{a l g}^{*}\left(P_{d}(\Gamma), \mathcal{A}\right)$ consisting of all elements whose supports are contained in $O(d) \times O(d)$. Define $C^{*}\left(P_{d}(\Gamma), \mathcal{A}\right)_{O}$ to be the norm closure of $C_{\text {alg }}^{*}\left(P_{d}(\Gamma), \mathcal{A}\right)_{O}$. We can similarly define $C_{L}^{*}\left(P_{d}(\Gamma), \mathcal{A}\right)_{O}$.

Lemma 6.2. Let $O$ and $O^{\prime}$ be open subsets of $\mathbb{R}_{+} \times H$. If $O \subseteq O^{\prime}$, then $C^{*}\left(P_{d}(\Gamma), \mathcal{A}\right)_{O}$ and $C_{L}^{*}\left(P_{d}(\Gamma), \mathcal{A}\right)_{O}$ are respectively closed, two sided ideals of $C^{*}\left(P_{d}(\Gamma), \mathcal{A}\right)_{O^{\prime}}$ and $C_{L}^{*}\left(P_{d}(\Gamma), \mathcal{A}\right)_{O^{\prime}}$.

The proof of Lemma 6.2 is straightforward and is therefore omitted. 
Lemma 6.3. Let $B(r)=\left\{(t, h) \in \mathbb{R}_{+} \times H: t^{2}+\|h\|^{2}<r^{2}\right\}$ for each $r>0$; let $X_{i}$ and $X_{i}^{\prime}$ be subsets of $\Gamma$ for all $1 \leq i \leq i_{0}$. If $O_{r}=\cap_{i=1}^{i_{0}}\left(\cup_{\gamma \in X_{i}} U_{\gamma, x_{0}} B(r)\right)$ and $O_{r}^{\prime}=$ $\cap_{i=1}^{i_{0}}\left(\cup_{\gamma \in X_{i}^{\prime}} U_{\gamma, x_{0}} B(r)\right)$, then for each $r_{0}>0$ we have

$$
\begin{gathered}
(1) \lim _{r<r_{0}, r \rightarrow r_{0}} C^{*}\left(P_{d}(\Gamma), \mathcal{A}\right)_{O_{r}}+\lim _{r<r_{0}, r \rightarrow r_{0}} C^{*}\left(P_{d}(\Gamma), \mathcal{A}\right)_{O_{r}^{\prime}} \\
=\lim _{r<r_{0}, r \rightarrow r_{0}} C^{*}\left(P_{d}(\Gamma), \mathcal{A}\right)_{O_{r} \cup O_{r}^{\prime}}, \\
\lim _{r<r_{0}, r \rightarrow r_{0}} C_{L}^{*}\left(P_{d}(\Gamma), \mathcal{A}\right)_{O_{r}}+\lim _{r<r_{0}, r \rightarrow r_{0}} C_{L}^{*}\left(P_{d}(\Gamma), \mathcal{A}\right)_{O_{r}^{\prime}} \\
=\lim _{r<r_{0}, r \rightarrow r_{0}} C_{L}^{*}\left(P_{d}(\Gamma), \mathcal{A}\right)_{O_{r} \cup O_{r}^{\prime}} ; \\
\lim _{r<r_{0}, r \rightarrow r_{0}}\left(C^{*}\left(P_{d}(\Gamma), \mathcal{A}\right)_{O_{r}} \cap C^{*}\left(P_{d}(\Gamma), \mathcal{A}\right)_{O_{r}^{\prime}}\right)=\lim _{r<r_{0}, r \rightarrow r_{0}} C^{*}\left(P_{d}(\Gamma), \mathcal{A}\right)_{O_{r} \cap O_{r}^{\prime}}, \\
\lim _{r<r_{0}, r \rightarrow r_{0}}\left(C_{L}^{*}\left(P_{d}(\Gamma), \mathcal{A}\right)_{O_{r}} \cap C_{L}^{*}\left(P_{d}(\Gamma), \mathcal{A}\right)_{O_{r}^{\prime}}\right)=\lim _{r<r_{0}, r \rightarrow r_{0}} C_{L}^{*}\left(P_{d}(\Gamma), \mathcal{A}\right)_{O_{r} \cap O_{r}^{\prime}} .
\end{gathered}
$$

Proof. We shall only prove the first identity. The rest of the identities can be proved in a similar way. It is enough to show that

$$
\lim _{r<r_{0}, r \rightarrow r_{0}} C_{a l g}^{*}\left(P_{d}(\Gamma), \mathcal{A}\right)_{O_{r} \cup O_{r}^{\prime}} \subseteq \lim _{r<r_{0}, r \rightarrow r_{0}} C_{a l g}^{*}\left(P_{d}(\Gamma), \mathcal{A}\right)_{O_{r}}+\lim _{r<r_{0}, r \rightarrow r_{0}} C^{*}\left(P_{d}(\Gamma), \mathcal{A}\right)_{O_{r}^{\prime}} .
$$

Given $T \in C_{\text {alg }}^{*}\left(P_{d}(\Gamma), \mathcal{A}\right)_{O_{r} \cup O_{r}^{\prime}}$ for some $r>0$, there exists $R>0$ such that

$$
\operatorname{support}(T(x, y)) \subseteq \cap_{i=1}^{i_{0}}\left(\cup_{\gamma \in X_{i} \cup X_{i}^{\prime}, d(\gamma, x) \leq R} U_{\gamma, x} B(r)\right)
$$

for all $x, y \in \Gamma_{d}$.

For each $k \in \mathbb{N}$, let $f_{r, k}$ be an even function in $\mathcal{S}$ such that (1) $0 \leq f_{r, k} \leq 1$, $\operatorname{support}\left(f_{r, k}\right) \subseteq\left(-r-\frac{1}{k}, r+\frac{1}{k}\right),\left.f_{r, k}\right|_{\left(-r-\frac{1}{2 k}, r+\frac{1}{2 k}\right)}=1 ;(2) f_{r, k}$ is differentiable and its derivative function is continuous. Let $g_{r, k}=\beta\left(f_{r, k}\right)$, where $\beta$ is the $*$ homomorphism: $\mathcal{S}=\mathcal{A}(0) \rightarrow \mathcal{A}(V)$, induced by the inclusion of the zero dimensional space 0 into $V$. Note that $g_{r, k} \in C_{0}\left(\mathbb{R}_{+} \times H\right)$. 
Choose $k \in \mathbb{N}$ such that $r+\frac{1}{k}<r_{0}$. For each $x \in \Gamma_{d}$, define $g_{x}$ and $g_{x}^{\prime}$ in $C_{0}\left(\mathbb{R}_{+} \times H\right)$ by:

$$
\begin{aligned}
& g_{x}=\frac{\prod_{i=1}^{i_{0}}\left(\sum_{\gamma \in X_{i}, d(\gamma, x) \leq R} U_{\gamma, x}^{-1}\left(g_{r, 2 k}\right)\right)}{\prod_{i=1}^{i_{0}}\left(\sum_{\gamma \in X_{i}, d(\gamma, x) \leq R} U_{\gamma, x}^{-1}\left(g_{r, k}\right)\right)+\prod_{i=1}^{i_{0}}\left(\sum_{\gamma \in X_{i}^{\prime}, d(\gamma, x) \leq R} U_{\gamma, x}^{-1}\left(g_{r, k}\right)\right)}, \\
& g_{x}^{\prime}=\frac{\prod_{i=1}^{i_{0}}\left(\sum_{\gamma \in X_{i}^{\prime}, d(\gamma, x) \leq R} U_{\gamma, x}^{-1}\left(g_{r, 2 k}\right)\right)}{\prod_{i=1}^{i_{0}}\left(\sum_{\gamma \in X_{i}, d(\gamma, x) \leq R} U_{\gamma, x}^{-1}\left(g_{r, k}\right)\right)+\prod_{i=1}^{i_{0}}\left(\sum_{\gamma \in X_{i}^{\prime}, d(\gamma, x) \leq R} U_{\gamma, x}^{-1}\left(g_{r, k}\right)\right)} .
\end{aligned}
$$

Define $T_{1}$ and $T_{2}$ in $C^{*}\left(P_{d}(\Gamma), \mathcal{A}\right)$ by: $T_{1}(x, y)=g_{x} T(x, y), T_{2}(x, y)=g_{x}^{\prime} T(x, y)$. We have

$$
T=T_{1}+T_{2}
$$

By the properties of $g_{x}$ and $g_{x}^{\prime}$, and the bounded geometry property of $\Gamma$, it is not difficult to verify that

$$
\begin{aligned}
& T_{1} \in \lim _{r<r_{0}, r \rightarrow r_{0}} C_{a l g}^{*}\left(P_{d}(\Gamma), \mathcal{A}\right)_{O_{r}}, \\
& T_{2} \in \lim _{r<r_{0}, r \rightarrow r_{0}} C_{a l g}^{*}\left(P_{d}(\Gamma), \mathcal{A}\right)_{O_{r}^{\prime}} .
\end{aligned}
$$

QED

Let $e$ be the evaluation homomorphism from $C_{L}^{*}\left(P_{d}(\Gamma), \mathcal{A}\right)$ to $C^{*}\left(P_{d}(\Gamma), \mathcal{A}\right)$ defined by:

$$
e(g)=g(0)
$$

Lemma 6.4. If $O$ is the union of a family of open subsets $\left\{O_{i}\right\}_{i \in I}$ in $\mathbb{R}_{+} \times H$ such that

(1) $O_{i} \cap O_{j}=\emptyset$ if $i \neq j$;

(2) $\exists r>0, \gamma_{i} \in \Gamma$ such that $U_{x_{0}, \gamma_{i}} O_{i} \subseteq B(r)$ for all $i$, where $B(r)=\{(t, h) \in$ $\left.\mathbb{R}_{+} \times H: t^{2}+\|h\|^{2}<r^{2}\right\}$

then

$$
e_{*}: \lim _{d \rightarrow \infty} K_{*}\left(C_{L}^{*}\left(P_{d}(\Gamma), \mathcal{A}\right)_{O}\right) \rightarrow \lim _{d \rightarrow \infty} K_{*}\left(C^{*}\left(P_{d}(\Gamma), \mathcal{A}\right)_{O}\right),
$$

is an isomorphism.

Proof. Let $\mathcal{A}(V)_{O}$ be the $C^{*}$ subalgebra of $\mathcal{A}(V)$ generated by elements whose supports are contained in $O$. The support of an element $\sum a_{x}[x]$ in $E$ ( $E$ is as in Definition 5.2) is 
defined to be

$$
\left\{(u, x) \in\left(\mathbb{R}_{+} \times H\right) \times \Gamma_{d}: a_{x}(u) \neq 0\right\} .
$$

Let $E_{O(d)}$ be the closure of the set of all elements in $E$ whose supports are contained in $O(d)$, where $O(d)$ is as in the definition of $C_{a l g}^{*}\left(P_{d}(\Gamma), \mathcal{A}\right)_{O} . E_{O(d)}$ is a Hilbert module over $\mathcal{A}(V)_{O} \widehat{\otimes} K . \quad C^{*}\left(P_{d}(\Gamma), \mathcal{A}\right)_{O}$ has a faithful representation on $E_{O(d)}$. We have a decomposition:

$$
E_{O(d)}=\bigoplus_{i \in I} E_{O_{i}(d)}
$$

where $O_{i}(d)$ is defined in way similar to the definition of $O(d)$. By the uniform embedding property, each element $a \in C_{\text {alg }}^{*}\left(P_{d}(\Gamma), \mathcal{A}\right)_{O}$ has a corresponding decomposition:

$$
a=\bigoplus_{i \in I} a_{i}
$$

such that there exists $R>0$ for which $a_{i}$ is supported on $O_{i}(d, R) \times O_{i}(d, R)$ for all $i$, where $O_{i}(d, R)=\left\{\left(U_{x_{0}, x} u, x\right): u \in O_{i}, x \in \Gamma_{d}, d\left(x, \gamma_{i}\right) \leq R\right\}$.

Hence $a_{i}$ lives in the image of the injective homomorphism from $B\left(E_{O_{i}(d, R)}\right)$ to $B\left(E_{O_{i}(d)}\right)$ :

$$
\psi_{i}: b \rightarrow\left(\begin{array}{ll}
b & 0 \\
0 & 0
\end{array}\right)
$$

with respect to the decomposition

$$
E_{O_{i}(d)}=E_{O_{i}(d, R)} \oplus E_{O_{i}(d, R)}^{\prime}
$$

for some Hilbert submodule $E_{O_{i}(d, R)}^{\prime}$ of $E_{O_{i}(d)}$ (such Hilbert submodule exists in this case).

Let $E_{i}$ be the Hilbert module over $\mathcal{A}(V)_{O_{i}} \widehat{\otimes} K$ defined by:

$$
E_{i}=\left(\mathcal{A}(V)_{O_{i}} \widehat{\otimes} K\right) \widehat{\otimes} \ell^{2}\left(\left\{x \in \Gamma_{d}: d\left(x, \gamma_{i}\right) \leq R\right\}\right)
$$

Let $I_{i}$ be the isometry from $E_{O_{i}(d, R)}$ to $E_{i}$ defined by:

$$
I_{i}: \sum a_{x}[x] \rightarrow \sum U_{x_{0}, x}\left(a_{x}\right) \delta_{x}
$$


where $\delta_{x}$ is the Dirac function at $x$.

Note that $B\left(E_{i}\right)$, the $C^{*}$ algebra of all module homomorphisms from $E_{i}$ to $E_{i}$ for which there is an adjoint module homomorphism, can be identified with

$$
\mathcal{A}(V)_{O_{i}} \widehat{\otimes} K \widehat{\otimes} B\left(l^{2}\left(\left\{x \in P_{d}: d\left(x, \gamma_{i}\right) \leq R\right\}\right)\right)
$$

Using this identification it is not difficult to verify that the map:

$$
a \rightarrow \bigoplus_{i \in I} I_{i} a_{i} I_{i}^{-1}
$$

gives a $*$ isomorphism from $C^{*}\left(P_{d}(\Gamma), \mathcal{A}\right)_{O}$ to the $C^{*}$ subalgebra of

$$
\lim _{R \rightarrow \infty}\left(\bigoplus_{i \in I}\left(\mathcal{A}(V)_{O_{i}} \widehat{\otimes} C^{*}\left(\left\{x \in \Gamma_{d}: d\left(x, \gamma_{i}\right) \leq R\right\}\right)\right)\right)
$$

generated by elements $\bigoplus_{i \in I} b_{i}$ such that

(1) $b_{i} \in \mathcal{A}(V)_{O_{i}} \widehat{\otimes} C^{*}\left(\left\{x \in \Gamma_{d}: d\left(x, \gamma_{i}\right) \leq R\right\}\right)$ for some $R>0$ and all $i \in I$;

(2) there exists a constant $C_{0}$ for which $\left\|b_{i}\right\| \leq C_{0}$ for all $i \in I$;

(3) there exist $N>0, c_{0}>0$ such that, for each $i$, there is $b_{i}^{\prime} \in \mathcal{A}\left(W_{N}\left(\gamma_{i}\right)\right) \widehat{\otimes} C^{*}(\{x \in$ $\left.\left.\Gamma_{d}: d\left(x, \gamma_{i}\right) \leq R\right\}\right)$ for which $U_{x_{0}, \gamma_{i}}\left(\left(\beta_{N}\left(\gamma_{i}\right)\right)\left(b_{i}^{\prime}\right)\right)=b_{i}, D_{Y}\left(b_{i}^{\prime}\right)$ exists in $\mathcal{A}\left(W_{N}\left(\gamma_{i}\right)\right) \widehat{\otimes} C^{*}(\{x \in$ $\left.\left.\Gamma_{d}: d\left(x, \gamma_{i}\right) \leq R\right\}\right)$ and $\left\|D_{Y}\left(b_{i}^{\prime}\right)\right\| \leq c_{0}$ for all $Y=(s, h) \in \mathbb{R}_{+} \times W_{N}\left(\gamma_{i}\right)$ satisfying $\|Y\| \leq 1$, where $\beta_{N}\left(\gamma_{i}\right)$ is the $*$ homomorphism: $\mathcal{A}\left(W_{N}\left(\gamma_{i}\right)\right) \widehat{\otimes} C^{*}\left(\left\{x \in \Gamma_{d}: d\left(x, \gamma_{i}\right) \leq\right.\right.$ $R\}) \rightarrow \mathcal{A}(V) \widehat{\otimes} C^{*}\left(\left\{x \in \Gamma_{d}: d\left(x, \gamma_{i}\right) \leq R\right\}\right)$, induced by the inclusion of $W_{N}\left(\gamma_{i}\right)$ into $V$.

Similarly $C_{L}^{*}\left(P_{d}(\Gamma), \mathcal{A}\right)_{O}$ is $*$ isomorphic to the $C^{*}$ subalgebra of

$$
\lim _{R \rightarrow \infty}\left(\bigoplus_{i \in I}\left(\mathcal{A}(V)_{O_{i}} \widehat{\otimes} C_{L}^{*}\left(\left\{x \in \Gamma_{d}: d\left(x, \gamma_{i}\right) \leq R\right\}\right)\right)\right)
$$

generated by elements $\bigoplus_{i \in I} b_{i}$ such that

(1) $b_{i} \in \mathcal{A}(V)_{O_{i}} \widehat{\otimes} C_{L}^{*}\left(\left\{x \in \Gamma_{d}: d\left(x, \gamma_{i}\right) \leq R\right\}\right)$ for some $R>0$ and all $i \in I$;

(2) there exists a constant $C_{0}$ such that $\left\|b_{i}\right\| \leq C_{0}$ for all $i \in I$; 
(3) there exists a bounded function $c(t)$ on $\mathbb{R}_{+}$for which

$$
\lim _{t \rightarrow \infty} c(t)=0 \text { and } \sup \left\{d(x, y):(x, y) \in \operatorname{supp}\left(b_{i}(t)\right)\right\} \leq c(t)
$$

where $\operatorname{supp}\left(b_{i}(t)\right)$ is defined to be the complement (in $\left\{x \in \Gamma_{d}: d\left(x, \gamma_{i}\right) \leq R\right\} \times\left\{x \in \Gamma_{d}\right.$ : $\left.\left.d\left(x, \gamma_{i}\right) \leq R\right\}\right)$ of all $\left(\gamma, \gamma^{\prime}\right)$ such that

$$
<\left(b_{i}(t)\right)\left(a \widehat{\otimes} \delta_{\gamma}\right), a^{\prime} \widehat{\otimes} \delta_{\gamma^{\prime}}>=0
$$

for all $a, a^{\prime} \in \mathcal{A}(V)_{O_{i}} \widehat{\otimes} K$;

(4) there exist $N>0, c_{0}>0$ such that, for each $i$, there is $b_{i}^{\prime} \in \mathcal{A}\left(W_{N}\left(\gamma_{i}\right)\right) \widehat{\otimes} C_{L}^{*}(\{x \in$ $\left.\left.\Gamma_{d}: d\left(x, \gamma_{i}\right) \leq R\right\}\right)$ for which $U_{x_{0}, \gamma_{i}}\left(\left(\beta_{N}\left(\gamma_{i}\right)\right)\left(b_{i}^{\prime}\right)\right)=b_{i}, D_{Y}\left(b_{i}^{\prime}\right)$ exists in $\mathcal{A}\left(W_{N}\left(\gamma_{i}\right)\right) \widehat{\otimes} C_{L}^{*}(\{x \in$ $\left.\left.\Gamma_{d}: d\left(x, \gamma_{i}\right) \leq R\right\}\right)$ and $\left\|D_{Y}\left(b_{i}^{\prime}\right)\right\| \leq c_{0}$ for $Y=(s, h) \in \mathbb{R}_{+} \times W_{N}\left(\gamma_{i}\right)$ satisfying $\|Y\| \leq 1$,

Now Lemma 6.4 follows from the above facts, Theorem 4.2, and its proof in [26] (c.f. a notational correction in [27], page 332). QED

Lemma 6.5. Let $B(r)=\left\{(t, h) \in \mathbb{R}_{+} \times H: t^{2}+\|h\|^{2}<r^{2}\right\}$ for some $r>0$. If $\Gamma$ has bounded geometry, then there exists an integer $l_{0}$ such that if $\bigcap_{k=1}^{\ell} U_{\gamma_{k}, x_{0}} B(r) \neq \emptyset$ for distinct elements $\gamma_{k}$ in $\Gamma$, then $\ell \leq l_{0}$.

Proof. $\bigcap_{k=1}^{\ell} U_{\gamma_{k}, x_{0}} B(r) \neq \emptyset$ implies that

$$
\bigcap_{k=1}^{\ell} U_{\gamma_{k}, \gamma_{1}} B(r) \neq \emptyset .
$$

Hence there exists $R>0$ such that $d\left(\gamma_{k}, \gamma_{1}\right) \leq R$, where $R$ depends only on $r$. This, together with the bounded geometry property of $\Gamma$, implies Lemma 6.5. QED

Theorem 6.6. If $\Gamma$ has bounded geometry, then $e_{*}$ is an isomorphism from $\lim _{d \rightarrow \infty}\left(C_{L}^{*}\left(P_{d}(\Gamma), \mathcal{A}\right)\right)$ to $\lim _{d \rightarrow \infty} K_{*}\left(C^{*}\left(P_{d}(\Gamma), \mathcal{A}\right)\right)$.

Proof. Let $B(r)=\left\{(t, h) \in \mathbb{R}_{+} \times H: t^{2}+\|h\|^{2}<r^{2}\right\}$. We define $O_{r}$ by:

$$
O_{r}=\bigcup_{\gamma \in \Gamma} U_{\gamma, x_{0}} B(r)
$$


We have

$$
\begin{aligned}
& C_{L}^{*}\left(P_{d}(\Gamma), \mathcal{A}\right)=\lim _{r \rightarrow \infty} C_{L}^{*}\left(P_{d}(\Gamma), \mathcal{A}\right)_{O_{r}}, \\
& C^{*}\left(P_{d}(\Gamma), \mathcal{A}\right)=\lim _{r \rightarrow \infty} C^{*}\left(P_{d}(\Gamma), \mathcal{A}\right)_{O_{r}} .
\end{aligned}
$$

Hence it is enough to show that $e_{*}$ is an isomorphism from $\lim _{d \rightarrow \infty} K_{*}\left(\lim _{r<r_{0}, r \rightarrow r_{0}} C_{L}^{*}\left(P_{d}(\Gamma), \mathcal{A}\right)_{O_{r}}\right)$ to $\lim _{d \rightarrow \infty} K_{*}\left(\lim _{r<r_{0}, r \rightarrow r_{0}} C^{*}\left(P_{d}(\Gamma), \mathcal{A}\right)_{O_{r}}\right)$ for every $r_{0}>0$. By Lemma 6.5 , for each $r_{0}>0$, there exist finitely many subsets $\left\{I_{k}\right\}_{k=1}^{k_{0}}$ of $\Gamma$ such that $O_{r}=\bigcup_{k=1}^{k_{0}} O_{r, k}$ for all $r<r_{0}$, where each $O_{r, k}$ is the disjoint union of $\left\{U_{\gamma, x_{0}} B(r)\right\}_{\gamma \in I_{k}}$ for all $r<r_{0}$. Now Theorem 6.6 follows from Lemmas 6.4, 6.2, 6.3, and a Mayer-Vietoris sequence argument. QED

\section{Proof of the main theorem}

In this section, we shall prove the main theorem of this paper.

We shall first recall certain infinite dimensional elliptic operators introduced by Higson, Kasparov and Trout [15].

Let $V$ be the countably infinite dimensional Euclidean space as in Section 5. Denote by $\mathcal{H}_{a}$ the Hilbert space of square integrable functions from $V_{a}$ into Cliff $\left(V_{a}^{0}\right)$. If $V_{a} \subset V_{b}$, then there exists an isomorphism:

$$
\mathcal{H}_{b} \cong \mathcal{H}_{b a} \widehat{\otimes} \mathcal{H}_{a}
$$

where $\mathcal{H}_{b a}$ is the Hilbert space associated to $V_{b a}^{0}$. Let $\xi_{0} \in \mathcal{H}_{b a}$ be the unit vector defined by:

$$
\xi_{0}\left(v_{b a}\right)=\pi^{-n_{b a} / 4} \exp \left(-\frac{1}{2}\left\|v_{b a}\right\|^{2}\right)
$$

where $n_{b a}=\operatorname{dim}\left(V_{b a}^{0}\right)$. We consider $\mathcal{H}_{a}$ as included in $\mathcal{H}_{b}$ via the isometry $\xi \rightarrow \xi_{0} \widehat{\otimes} \xi$. We define

$$
\mathcal{H}=\underset{\lim }{\longrightarrow} \mathcal{H}_{a}
$$

where the Hilbert space direct limit is taken over the direct system of finite dimensional affine subspaces of $V$. 
Let $s=\lim _{a}$ be the algebraic direct limit of the Schwartz subspaces $s_{a} \subset \mathcal{H}_{a}$. If $V_{a} \subset V$ is a finite dimensional affine subspace, we define the Dirac operator $D_{a}$, an unbounded operator on $\mathcal{H}$ with domain $s$, to be:

$$
D_{a} \xi=\sum_{i=1}^{n}(-1)^{\operatorname{deg} \xi} \frac{\partial \xi}{\partial x_{i}} v_{i}
$$

where $\left\{v_{1}, \ldots, v_{n}\right\}$ is an orthonormal basis for $V_{a}^{0}$ and $\left\{x_{1}, \ldots, x_{n}\right\}$ are the dual coordinates to $\left\{v_{1}, \ldots, v_{n}\right\}$. If $V_{a}$ is a linear subspace, then we define the Clifford operator by:

$$
C_{a} \xi=\sum_{i=1}^{n} x_{i} v_{i} \xi
$$

Let $V_{n}(x)=W_{n+1}(x) \ominus W_{n}(x)$ if $n \geq 1, V_{0}(x)=W_{1}(x)$, where $W_{n}(x)$ is as in Section 5 . We have the algebraic decomposition:

$$
V=W(x)=V_{0}(x) \oplus V_{1}(x) \oplus \cdots \oplus V_{n}(x) \oplus \cdots
$$

For each $n$, define an unbounded operator $B_{n, t}(x)$ on $\mathcal{H}$ associated to the above decomposition by:

$$
B_{n, t}(x)=t_{0} D_{0}+t_{1} D_{1}+\cdots+t_{n-1} D_{n-1}+t_{n}\left(D_{n}+C_{n}\right)+t_{n+1}\left(D_{n+1}+C_{n+1}\right)+\cdots
$$

where $t_{j}=1+t^{-1} j$. By Lemma 5.8 in [15], $B_{n, t}(x)$ is essentially selfadjoint.

For each $s \in[0, \infty)$, we define $C_{a l g}^{*}\left(P_{d}(\Gamma), K(s)\right)$ to be the algebra of all functions $T$ on $\Gamma_{d} \times \Gamma_{d}$ such that

(1) $T(x, y) \in K(\mathcal{H}) \widehat{\otimes} K$ for all $x, y \in \Gamma_{d}$, where $K(\mathcal{H})$ is the algebra of all compact operators acting on $\mathcal{H}$;

(2) $\exists M>0$ and $L>0$ such that $\|T(x, y)\| \leq M$ for all $x, y \in \Gamma_{d}$, and for each $y \in \Gamma_{d}$, $\#\left\{x \in \Gamma_{d}: T(x, y) \neq 0\right\} \leq L, \#\left\{x \in \Gamma_{d}: T(y, x) \neq 0\right\} \leq L ;$

(3) $\exists r>0$ such that if $d(x, y)>r$, then $T(x, y)=0$. 
The product structure on $C_{\text {alg }}^{*}\left(P_{d}(\Gamma), K(s)\right)$ is defined by:

$$
\left(T_{1} \cdot T_{2}\right)(x, y)=\sum_{z \in \Gamma_{d}} T_{1}(x, z) U_{x, z}(s)\left(T_{2}(z, y)\right)
$$

where $U_{x, z}(s)\left(T_{2}(z, y)\right)=\left(U_{x, z}(s) \widehat{\otimes} 1\right) T_{2}(z, y)\left(U_{x, z}^{-1}(s) \widehat{\otimes} 1\right), U_{x, z}(s)$ is the unitary operator acting on $\mathcal{H}$ induced by the unitary operator $U_{x, z}(s)$ on $V$ defined by: $\left(U_{x, z}(s)\right) h=$ $h+s(f(x)-f(z))$ for all $h \in V$.

Let $E=\ell^{2}\left(\Gamma_{d}\right) \widehat{\otimes} \mathcal{H} \widehat{\otimes} H_{0}$, where $H_{0}$ is a separable and infinite dimensional Hilbert space with a faithful $*$ representation of $K$.

$C_{a l g}^{*}\left(P_{d}(\Gamma), K(s)\right)$ acts on $E$ by:

$$
T\left(\delta_{x} \widehat{\otimes} h \widehat{\otimes} h_{0}\right)=\sum_{y \in \Gamma_{d}} \delta_{y} \widehat{\otimes} T(y, x)\left(U_{y, x}(s) h \widehat{\otimes} h_{0}\right)
$$

for all $x \in \Gamma_{d}, h \in \mathcal{H}, h_{0} \in H_{0}$.

Definition 7.1: $C^{*}\left(P_{d}(\Gamma), K(s)\right)$ is defined to be the operator norm closure of $C_{\text {alg }}^{*}\left(P_{d}(\Gamma), K(s)\right)$ with respect to the above $*$ representation.

Let $\phi_{s}$ be the map from $C_{a l g}^{*}\left(P_{d}(\Gamma), K(s)\right)$ to $C_{a l g}^{*}\left(P_{d}(\Gamma), K(0)\right)$ defined by:

$$
\left(\phi_{s}(T)\right)(x, y)=U_{x_{0}, x}(s) T(x, y) U_{x_{0}, x}^{-1}(s)
$$

for all $T \in C_{a l g}^{*}\left(P_{d}(\Gamma), K(s)\right)$.

Lemma 7.2. $\phi_{s}$ extends to a* isomorphism from $C^{*}\left(P_{d}(\Gamma), K(s)\right)$ to $C^{*}\left(P_{d}(\Gamma), K(0)\right)$.

The proof of the above lemma is straightforward and is therefore omitted.

For each $f \in S, g \in \mathcal{C}\left(V_{0}(x) \oplus \cdots \oplus V_{n-1}(x)\right), k \in K$, we define

$$
\alpha_{t}^{n}(x):(f \widehat{\otimes} g) \widehat{\otimes} k \rightarrow \phi_{t}\left(f_{t}\left(B_{n, t}(x)\right) \pi\left(g_{t}\right) \widehat{\otimes} k\right)
$$

where $f_{t}(s)=f\left(t^{-1} s\right)$ for all $t>0$ and $s \in \mathbb{R}, g_{t}(v)=g\left(t^{-1} v\right)$ for all $t>0$ and $v \in$ $V_{0}(x) \oplus \cdots \oplus V_{n-1}(x), \pi\left(g_{t}\right)$ acts on $\mathcal{H}$ by pointwise multiplication, and $\phi_{t}$ is as in Lemma 7.2 . 
For each $T \in C_{a l g}^{*}\left(P_{d}(\Gamma), \mathcal{A}\right)$, we define $\alpha_{t}(T)$ in $C^{*}\left(P_{d}(\Gamma), K(0)\right)$ by:

$$
\left(\alpha_{t}(T)\right)(x, y)=\left(\alpha_{t}^{N}(x)\right)\left(T_{1}(x, y)\right)
$$

where $N$ is some integer such that there exists $T_{1}(x, y) \in \mathcal{A}\left(W_{N}(x)\right) \widehat{\otimes} K$ for which $\left(\beta_{N}(x)\right)\left(T_{1}(x, y)\right)=$ $T(x, y)$ for every $x, y \in \Gamma_{d}$.

Lemma 7.3. $\alpha$ extends to an asymptotic morphism from $C^{*}\left(P_{d}(\Gamma), \mathcal{A}\right)$ to $C^{*}\left(P_{d}(\Gamma), K(0)\right)$.

Proof. $\forall T \in C_{\text {alg }}^{*}\left(P_{d}(\Gamma), \mathcal{A}\right)$, define

$$
\|T\|_{\max }=\sup _{\psi}\|\psi(T)\|
$$

where the sup is taken over all $*$ representation $\psi$ of $C_{\text {alg }}^{*}\left(P_{d}(\Gamma), \mathcal{A}\right)$.

Let $C_{\max }^{*}\left(P_{d}(\Gamma), \mathcal{A}\right)$ be the completion of $C_{\text {alg }}^{*}\left(P_{d}(\Gamma), \mathcal{A}\right)$ with respect to the norm \|\|$_{\max }$

By the proof of Lemma 2.9 and Lemma 5.12 in [15], condition (4) of the definition of $C_{a l g}^{*}\left(P_{d}(\Gamma), \mathcal{A}\right)$, and Lemma 7.2 , we know that $\alpha$ extends to an asymptotic morphism from $C_{\max }^{*}\left(P_{d}(\Gamma), \mathcal{A}\right)$ to $C^{*}\left(P_{d}(\Gamma), K(0)\right)$. But by the uniform embedding property we have $C^{*}\left(P_{d}(\Gamma), \mathcal{A}\right)=C_{\max }^{*}\left(P_{d}(\Gamma), \mathcal{A}\right)$. Hence $\alpha$ extends to an asymptotic morphism from $C^{*}\left(P_{d}(\Gamma), \mathcal{A}\right)$ to $C^{*}\left(P_{d}(\Gamma), K(0)\right)$. QED

We remark that the asymptotic morphism $\alpha$ is adapted from [14].

Let $C_{a l g}^{*}\left(P_{d}(\Gamma)\right)$ be the algebra of functions $T$ on $\Gamma_{d} \times \Gamma_{d}$ such that

(1) $T(x, y) \in K$ for all $x, y \in \Gamma_{d}$;

(2) $\exists M>0$ and $L>0$ such that $\|T(x, y)\| \leq M$ for all $x, y \in \Gamma_{d}, \#\left\{x \in \Gamma_{d}\right.$ : $T(x, y) \neq 0\} \leq L, \#\left\{x \in \Gamma_{d}: T(y, x) \neq 0\right\} \leq L$

(3) $\exists r>0$ such that if $d(x, y)>r$, then $T(x, y)=0$.

The product structure on $C_{a l g}^{*}\left(P_{d}(\Gamma)\right)$ is defined by:

$$
\left(T_{1} T_{2}\right)(x, y)=\sum_{z \in \Gamma_{d}} T_{1}(x, z) T_{2}(z, y)
$$


$C_{a l g}^{*}\left(P_{d}(\Gamma)\right)$ has a $*$ representation on $\ell^{2}\left(\Gamma_{d}\right) \otimes H_{0}$, where $H_{0}$ is a separable infinite dimensional Hilbert space. The operator norm completion of $C_{a l g}^{*}\left(P_{d}(\Gamma)\right)$ with respect to this $*$ representation is $*$ isomorphic to $C^{*}\left(P_{d}(\Gamma)\right)$ when $\Gamma$ has bounded geometry. Similarly we can define $C_{L, a l g}^{*}\left(P_{d}(\Gamma)\right)$, and the operator norm completion of $C_{L, a l g}^{*}\left(P_{d}(\Gamma)\right)$ is * isomorphic to $C_{L}^{*}\left(P_{d}(\Gamma)\right)$ when $\Gamma$ has bounded geometry.

For each $f \in S, T \in C_{\text {alg }}^{*}\left(P_{d}(\Gamma)\right)$, we define

$$
\beta_{t}(f \widehat{\otimes} T) \in C^{*}\left(P_{d}(\Gamma), \mathcal{A}\right)
$$

by:

$$
\beta_{t}(f \widehat{\otimes} T)(x, y)=\beta\left(f_{t}\right) \widehat{\otimes} T(x, y),
$$

where $f_{t}(s)=f\left(t^{-1} s\right)$ for all $t>0$ and $s \in \mathbb{R}$, and $\beta: \mathcal{S}=\mathcal{A}(0) \rightarrow \mathcal{A}(V)$, is the *-homomorphism associated to the inclusion of the zero-dimensional linear space 0 into $V$.

Lemma 7.4. $\beta_{t}$ extends to an asymptotic morphism from $\mathcal{S} \widehat{\otimes} C^{*}\left(P_{d}(\Gamma)\right)$ to $C^{*}\left(P_{d}(\Gamma), . . \mathcal{A}\right)$.

Proof. Note that $\beta(\mathcal{S})$ and $C^{*}\left(P_{d}(\Gamma)\right)$ embed in the multiplier algebra of $C^{*}\left(P_{d}(\Gamma), \mathcal{A}\right)$. For every $f \in \mathcal{S}$ and $T \in C^{*}\left(P_{d}(\Gamma)\right), \beta\left(f_{t}\right)$ asymptotically commutes with $T$ in the multiplier algebra of $C^{*}\left(P_{d}(\Gamma), \mathcal{A}\right)$, i.e. $\lim _{t \rightarrow \infty}\left(\left(\beta\left(f_{t}\right)\right) T-T\left(\beta\left(f_{t}\right)\right)\right)=0$. This fact, together with the nuclearity of $\mathcal{S}$, implies that $\beta_{t}$ extends to an asymptotic morphism from $\mathcal{S} \widehat{\otimes} C^{*}\left(P_{d}(\Gamma)\right)$ to $C^{*}\left(P_{d}(\Gamma), \mathcal{A}\right)$. QED

We remark that the asymptotic morphism $\beta$ is adapted from [14] and [15].

Note that $\alpha$ and $\beta$ induce homomorphisms:

$$
\begin{aligned}
& \alpha_{*}: K_{*}\left(C^{*}\left(P_{d}(\Gamma), \mathcal{A}\right)\right) \rightarrow K_{*}\left(C^{*}\left(P_{d}(\Gamma)\right)\right), \\
& \beta_{*}: K_{*}\left(C^{*}\left(P_{d}(\Gamma)\right)\right) \rightarrow K_{*}\left(C^{*}\left(P_{d}(\Gamma), \mathcal{A}\right)\right) .
\end{aligned}
$$


Lemma 7.5. $\alpha_{*} \circ \beta_{*}$ equals the identity homomorphism from $K_{*}\left(C^{*}\left(P_{d}(\Gamma)\right)\right)$ to $K_{*}\left(C^{*}\left(P_{d}(\Gamma)\right)\right)$.

Proof. For each $s \in(0,1]$, we can define $C^{*}\left(P_{d}(\Gamma), \mathcal{A}(s)\right)$ by replacing $U_{x, y}$ with $U_{x, y}(s)$ in the definition of $C^{*}\left(P_{d}(\Gamma), \mathcal{A}\right)$, where $\left(U_{x, y}(s)\right) h=h+s(f(x)-f(y))$ for all $h \in V$. We can similarly define asymptotic morphisms:

$$
\begin{gathered}
\beta(s): \mathcal{S} \widehat{\otimes} C^{*}\left(P_{d}(\Gamma)\right) \rightarrow C^{*}\left(P_{d}(\Gamma), \mathcal{A}(s)\right) \\
\alpha(s): C^{*}\left(P_{d}(\Gamma), \mathcal{A}(s)\right) \rightarrow C^{*}\left(P_{d}(\Gamma), K(0)\right) .
\end{gathered}
$$

It is not difficult to see that $(\alpha(s)) \circ(\beta(s))$ is a homotopy of asymptotic morphisms $(s \in$ $(0,1])$, and $\lim _{s \rightarrow 0^{+}}((\alpha(s)) \circ(\beta(s)))(a)$ equals $\gamma(a)$ for all $a \in \mathcal{S} \widehat{\otimes} C^{*}\left(P_{d}(\Gamma)\right)$, where $\gamma$ is the asymptotic morphism

$$
\gamma: \mathcal{S} \widehat{\otimes} C^{*}\left(P_{d}(\Gamma)\right) \rightarrow C^{*}\left(P_{d}(\Gamma), K(0)\right)
$$

defined by:

$$
\left(\gamma_{t}(f \widehat{\otimes} T)\right)(x, y)=f_{t}\left(B_{0, t}\right) \widehat{\otimes} T(x, y)
$$

for each $f \in \mathcal{S}, T \in C_{\text {alg }}^{*}\left(P_{d}(\Gamma)\right)$. Replacing $B_{0, t}$ with $s^{-1} B_{0, t}$ in the above definition of $\gamma$, for $0<s \leq 1$, we obtain a homotopy between $\gamma$ and the $*$ homomorphism: $f \widehat{\otimes} T \rightarrow$ $f(0) P \widehat{\otimes} T$, where $P$ is the projection onto the one-dimensional kernel of $B_{0, t}$ (c.f. Corollary 2.15 and the proof of Lemma 5.8 in [15]). Hence $\gamma_{*}$ equals the identity homomorphism. QED

We can similarly construct asymptotic morphisms

$$
\begin{gathered}
\alpha_{L}: C_{L}^{*}\left(P_{d}(\Gamma), \mathcal{A}\right) \rightarrow C_{L}^{*}\left(P_{d}(\Gamma), K(0)\right), \\
\beta_{L}: \mathcal{S} \widehat{\otimes} C_{L}^{*}\left(P_{d}(\Gamma)\right) \rightarrow C_{L}^{*}\left(P_{d}(\Gamma), \mathcal{A}\right),
\end{gathered}
$$

where $C_{L}^{*}\left(P_{d}(\Gamma), K(0)\right)$ is defined in a way similar to the definition of $C^{*}\left(P_{d}(\Gamma), K(0)\right)$. 
Lemma 7.6. $\left(\alpha_{L}\right)_{*} \circ\left(\beta_{L}\right)_{*}$ equals the identity homomorphism from $K_{*}\left(C_{L}^{*}\left(P_{d}(\Gamma)\right)\right.$ to $K_{*}\left(C_{L}^{*}\left(P_{d}(\Gamma)\right)\right.$

The proof of Lemma 7.6 is similar to the proof of Lemma 7.5 and is therefore omitted.

Proof of Theorem 1.1.

By Theorem 4.2, it is enough to show that

$$
e_{*}: \lim _{d \rightarrow \infty} K_{*}\left(C_{L}^{*}\left(P_{d}(\Gamma)\right)\right) \rightarrow \lim _{d \rightarrow \infty} K_{*}\left(C^{*}\left(P_{d}(\Gamma)\right)\right)
$$

is an isomorphism. Consider the following commuting diagram:

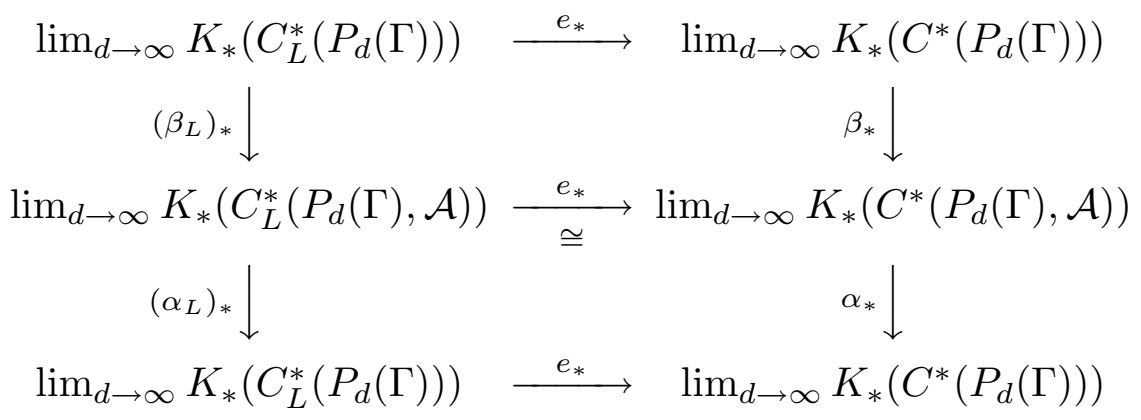

By Theorem 6.6 and Lemmas 7.5 and 7.6, the middle horizontal homomorphism, $\left(\beta_{L}\right)_{*} \circ$ $\left(\alpha_{L}\right)_{*}$ and $\beta_{*} \circ \alpha_{*}$ are identity homomorphisms. This fact, together with a diagram chasing argument, implies Theorem 1.1. QED

\section{References}

1. P. Baum and A. Connes, K-theory for discrete groups, Operator Algebras and Applications, (D. Evans and M. Takesaki, editors), Cambridge University Press (1989), $1-20$.

2. M.E.B. Bekka, P.A. Cherix and A. Vallete, Proper affine isometric actions of amenable groups, Novikov Conjectures, Index Theorems and Rigidity, Vol. 2, (S. Ferry, A. Ranicki and J. Rosenberg, editors), Cambridge University Press (1995), 1-4.

3. G. Carlsson and E.K. Pedersen, Controlled algebra and the Novikov conjectures for K and L theory, Topology 34 (1994), 731-758. 
4. A. Connes, Cyclic cohomology and transverse fundamental class of a foliation, Geometric Methods in Operator Algebras, (H. Araki and E.G. Effros, editors), Pitman Res. Notes Math., Vol. 123 (1996), 52-144.

5. A. Connes, Noncommutative Geometry, Academic Press, 1994.

6. A. Connes, M. Gromov and H. Moscovici, Group cohomology with Lipschitz control and higher signatures, Geometric and Functional Analysis, 3 (1993), 1-78.

7. A. Connes and H. Moscovici, Cyclic cohomology, the Novikov conjecture and hyperbolic groups, Topology 29 (1990), 345-388.

8. S. Ferry, A. Ranicki and J. Rosenberg, A history and survey of the Novikov conjecture, Novikov Conjectures, Index Theorems and Rigidity, Vol. 1, (S. Ferry, A. Ranicki and J. Rosenberg, editors), Cambridge University Press, (1995), 7-66.

9. S. Ferry and S. Weinberger, A coarse approach to the Novikov conjecture, Novikov Conjectures, Index Theorems and Rigidity, Vol. 2, (S. Ferry, A. Ranicki and J. Rosenberg, editors), Cambridge University Press, (1995), 146-163.

10. M. Gromov, Asymptotic invariants for infinite groups, Geometric Group Theory, (G. A. Niblo and M. A. Roller, editors), Cambridge University Press, (1993), 1-295.

11. M. Gromov, Problems (4) and (5), Novikov Conjectures, Index Theorems and Rigidity, Vol. 1, (S. Ferry, A. Ranicki and J. Rosenberg, editors), Cambridge University Press, (1995), 67 .

12. M. Gromov, Positive curvature, macroscopic dimension, spectral gaps and higher signatures, Functional Analysis on the eve of the 21st century, Vol. 2, Progr. Math. 132, (1996), 1-213.

13. E. Guentner, N. Higson and J. Trout, Equivariant E-theory, Preprint (1997).

14. N. Higson and G. G. Kasparov, Operator K-theory for groups which act properly and isometrically on Hilbert space, Electronic Research Announcements, AMS 3 (1997), 131-141. 
15. N. Higson, G. G. Kasparov and J. Trout, A Bott periodicity theorem for infinite dimensional Euclidean space, Advances in Math. 135 (1998), No. 1, 1-40.

16. N. Higson and J. Roe, On the coarse Baum-Connes conjecture, Novikov Conjectures, Index Theorems and Rigidity, Vol. 2, (S. Ferry, A. Ranicki and J. Rosenberg, editors), Cambridge University Press, (1995), 227-254.

17. N. Higson, J. Roe and G. Yu, A coarse Mayer-Vietoris principle, Math. Proc. Camb. Philos. Soc. 114 (1993), 85-97.

18. G. G. Kasparov, Equivariant KK-theory and the Novikov conjecture, Inventiones Mathematicae, 91 (1988), 147-201.

19. G. G. Kasparov and G. Skandalis, Groups acting properly on "bolic" spaces and the Novikov conjecture, Preprint (1998).

20. A. S. Miscenko, Homotopy invariants of non-simply connected manifolds, Izv. Akad. Nauk, SSSR 43 (1979), 831-859.

21. J. Roe, Coarse cohomology and index theory for complete Riemannian manifolds, Memoirs A.M.S., No. 104 (1993).

22. J. Roe, Index Theory, Coarse Geometry, and Topology of Manifolds, CBMS Regional Conf. Series in Math, Number 90, AMS (1996).

23. J. Rosenberg, $C^{*}$-algebras, positive scalar curvature and the Novikov Conjecture, Publ I.H.E.S., No. 58 (1983), 197-212.

24. Z. Sela, Uniform embeddings of hyperbolic groups in Hilbert spaces, Israel Journal of Mathematics 80 (1992), 171-181.

25. G. Yu, Coarse Baum-Connes conjecture, K-Theory 9 (1995), 199-221.

26. G. Yu, Localization algebras and the coarse Baum-Connes conjecture, K-Theory 11 (1997), 307-318.

27. G. Yu, The Novikov Conjecture for groups with finite asymptotic dimension, Annals of Mathematics, Vol. 147, 2 (1998), 325-355. 
Department of Mathematics

University of Colorado

Boulder, CO 80309-0395, USA

e-mail: gyu@euclid.colorado.edu 